\title{
LOWER SEMIMODULAR LIE ALGEBRAS
}

\author{
by V. R. VAREA
}

(Received 29th August 1997)

\begin{abstract}
This paper is concerned with the relationship between the properties of the subalgebra lattice $\mathcal{L}(L)$ of a Lie algebra $L$ and the structure of $L$. If the lattice $\mathcal{L}(L)$ is lower semimodular, then the Lie algebra $L$ is said to be lower semimodular. If a subalgebra $S$ of $L$ is a modular element in the lattice $\mathcal{L}(L)$, then $S$ is called a modular subalgebra of $L$. The easiest condition to ensure that $L$ is lower semimodular is that $\operatorname{dim} A / B=1$ whenever $B<A \leq L$ and $B$ is maximal in $A$ (Lie algebras satisfying this condition are called $s \chi$-algebras). Our aim is to characterize lower semimodular Lie algebras and s $\chi$-algebras, over any field of characteristic greater than three. Also, we obtain results about the influence of two solvable modular maximal subalgebras on the structure of the Lie algebra and some results on the structure of Lie algebras all of whose maximal subalgebras are modular.
\end{abstract}

1991 Mathematics subject classification: Primary 17B05, Secondary 17B50.

\section{Introduction}

A subalgebra $M$ of $L$ is called modular in $L$ if it is a modular element in the lattice of all subalgebras of $L$; that is, if for each subalgebra $S$ of $L$ the mapping

$$
\phi_{M, S}:[M: M \vee S] \ni T \rightarrow T \cap S \in[M \cap S: S]
$$

is a bijection. (Here, $X \vee Y$ denotes the subalgebra of $L$ generated by $X$ and $Y$.) Note that a maximal subalgebra $M$ of $L$ is modular if and only if $M \cap S$ is maximal in $S$ for every $S \leq L$ with $S \geq M$. If every maximal subalgebra of $L$ is modular, then $L$ is said to be an $M(1)$-algebra. A Lie algebra $L$ is called lower semimodular if the lattice of subalgebras of $L$ is lower semimodular (that is, if every subalgebra of $L$, including $L$ itself, is an $M(1)$-algebra).

For fields $F$ of characteristic zero, lower semimodular Lie algebras were classified by Gein [9] and $M(1)$-algebras with trivial Frattini subalgebra are classified in [17]. Gein proved that $L$ is lower semimodular if and only if $L=U \oplus P_{1} \oplus \cdots \oplus P_{r}$, where $U$ is supersolvable, $r \geq 0$ and the $P_{i}$ are mutually non-isomorphic three-dimensional simple Lie algebras, and also $r \leq 1$ when $\sqrt{F} \leq F$, and the $P_{i}$ are non-split when $\sqrt{F} \notin F$. In [17], it is proved that a Lie algebra $L$ is $M(1)$ if and only if $L / \operatorname{Fr}(L)$ is lower semimodular, where $\operatorname{Fr}(L)$ denotes the Frattini subalgebra of $L$ (that is, the intersection of the maximal subalgebras of $L$ ).

For fields of prime characteristic, Gein's result above fails. Indeed, certain 
Yermolaev algebras $Y$ satisfy $Y / \operatorname{Fr}(Y) \cong \operatorname{sl}(2)$ (see [23]), and we will see that such Lie algebras are lower semimodular, provided the ground field is algebraically closed of characteristic greater than three. However, we do not know any example of an $M(1)$-algebra with trivial Frattini subalgebra which is not lower semimodular.

The easiest examples of $M(1)$-algebras are the Lie algebras in which every maximal subalgebra has codimension one (these algebras are called $\chi$-algebras). So, the easiest condition to ensure that a Lie algebra $L$ is lower semimodular is that every subalgebra of $L$ (including $L$ itself) is a $\chi$-algebra (for short, Lie algebras satisfying this condition will be called $s \chi$-algebras). $\chi$-algebras $L$ such that $\phi(L)=0$ were classified by Towers in [15] and [16], where $\phi(L)$ denotes the largest ideal of $L$ contained in $\operatorname{Fr}(L)$. In [23] it is proved that for any algebraically closed field of characteristic greater than three, a nonsolvable Lie algebra $L$ is minimal nonsupersolvable (that is, every proper subalgebra of $L$ is supersolvable) if and only if $L / \phi(L) \cong \mathrm{sl}(2)$; so, such an algebra is in the class $s \chi$. (Note that, in characteristic zero, $L / \phi(L) \cong \operatorname{sl}(2)$ implies $L \cong \operatorname{sl}(2)$.) Also, in [23] there is given a method to construct Lie algebras $L$ such that $L / \phi(L) \cong \operatorname{sl}(2)$.

In Section 2, we study the effect that the existence of two distinct modular solvable maximal subalgebras causes in the structure of the algebra.

In Section 3, we obtain some results on $M(1)$-algebras which will be needed in the sequel. The structural problem of an arbitrary $M(1)$-algebra will remain open. For any field, we prove that if $L$ is $M(1)$ then $R(L)$ is supersolvable and $R(L) \cap L^{u} \leq \phi(L)$, where $L^{u}$ denotes the smallest ideal of $L$ such that $L / L^{u}$ is supersolvable.

In Section 4, we study s $\chi$-algebras over fields $F$ such that $\operatorname{char}(F) \neq 2$. When $\sqrt{F} \not F$, the supersolvable algebras are the only $\chi$-algebras. When $\sqrt{F} \leq F$, we prove that $L$ is an s $\chi$-algebra if and only if either $L$ is supersolvable or else $L / R(L) \cong \mathrm{sl}(2) \cong L^{\mu} / \phi\left(L^{u}\right)$ and every strongly solvable subalgebra is supersolvable (this last condition is superfluous when $F$ is algebraically closed).

In Section 5, we consider lower semimodular Lie algebras over fields $F$ such that $\operatorname{char}(F)>3$. A central role will be played by the simple Lie algebras all of whose subalgebras of dimension greater than one are simple (called supersimple). We will prove that the supersimple algebras are the only simple Lie algebras which might be lower semimodular, provided $\sqrt{F} \leq F$. A Lie algebra of dimension greater than one is supersimple if and only if it contains no subalgebras of dimension two ([18, Proposition 3.2]). For perfect fields of characteristic $\neq 2,3$, there are no supersimple Lie algebras other than the three-dimensional non-split simple (see [19, Lemma 1.1] and [10]). The only known example of a supersimple Lie algebra of dimension greater than three is that constructed by Gein in [10, Example 12] over a certain perfect field of characteristic three. This algebra is not lower semimodular, its proper subalgebras of dimension greater than one are three-dimensional non-split simple and it cannot be generated by two elements. By contrast, for fields of characteristic $>3$, a supersimple Lie algebra must be bi-generated and it cannot have any three-dimensional non-split simple subalgebra ([22, Proposition 3.1]). A special type of supersimple Lie algebras are the Lie algebras of dimension greater than two all of whose proper subalgebras are onedimensional (called $\mu$-algebras). 
In the case where $\sqrt{F} \notin F$, we obtain that a Lie algebra $L$ is lower semimodular if and only if $L / Z\left(L^{(\infty)}\right) \cong U \oplus P_{1} \oplus \cdots \oplus P_{r}$, where $U$ is supersolvable, $r \geq 0$, each $P_{i}$ is a lower semimodular supersimple Lie algebra and for $i \neq j$, none of the subalgebras of $P_{i}$ of dimension greater than one is isomorphic to a subalgebra of $P_{j}$. We wonder if $Z\left(L^{(\infty)}\right)=0$ when $L$ is lower semimodular. This has an affirmative answer in the case where $F$ is perfect.

In the case where $\sqrt{F} \leq F$ we need to impose the additional condition that there are no $\mu$-algebras of dimension greater than three over the field $F$. We obtain that the $s \chi$-algebras are the only lower semimodular Lie algebras.

As consequences of ours results, we have the following: (1) the classification of lower semimodular Lie algebras given by Gein [9] remains true whenever $F$ is perfect and

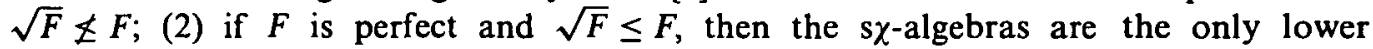
semimodular Lie algebras; (3) if $F$ is algebraically closed, then lower semimodular Lie algebras $L$ such that $L=L^{\prime}$ are precisely the Lie algebras $L$ such that $L / \phi(L) \cong \operatorname{sl}(2)$; and (4) if $F$ is finite, then the supersolvable algebras are the only lower semimodular Lie algebras.

Throughout, $L$ will denote a finite-dimensional Lie algebra over a field $F, Z(L)$ will be its centre, $R(L)$ its solvable radical, $\operatorname{Nil}(L)$ its nil-radical (that is, the largest nilpotent ideal of $L), \phi(L)$ its largest ideal contained in its Frattini subalgebra $\operatorname{Fr}(L), L^{\prime}$ its derived subalgebra, $L^{(\infty)}$ the last term in its derived series, $L^{\infty}$ the last term in its lower central series and $L^{u}$ its smallest ideal such that $L / L^{u}$ is supersolvable. Also, we will denote by $E_{L}(x)$ the Engel subalgebra of $L$ corresponding to the element $x$ of $L$ (so that $E_{L}(x)$ is the Fitting null-component of $L$ corresponding to the transformation $\operatorname{ad} x$ ). If $L^{\prime}$ is nilpotent, then the Lie algebra $L$ is said to be strongly solvable. It is wellknown that the class of strongly solvable Lie algebras is between the classes of supersolvable and solvable Lie algebras. For fields of characteristic zero, every solvable Lie algebra is strongly solvable (see [12] or [13]), whereas for algebraically closed fields, every strongly solvable Lie algebra is supersolvable (see [4]).

\section{Modular subalgebras and the structure of the Lie algebra}

There are many results in the literature on the effect that a modular subalgebra $M$ of a Lie algebra $L$ causes in the structure of the Lie algebra $L$ (see for instance $[2,10$, 11, 20 and 21]). If either $\operatorname{char}(F)=0$ or $L$ is solvable, then it is well-known that $M$ must be a quasi-ideal of $L$ (this means that $M \vee F x=M+F x$ for every $x \in L$ ), except when $\operatorname{dim} M / M_{L}=1$ and $L / M_{L}$ is three-dimensional non-split simple (here $M_{L}$ denotes the largest ideal of $L$ contained in $M$ ), see [2] and [20]. So, in those cases, the structure of the Lie algebra $L / M_{L}$ is known ([1, Theorem 3.6] and [2, Theorem 3.1]). However, in [21], it is shown that the standard maximal subalgebra of the simple Lie algebra of Cartan type $\mathcal{H}(2: \underline{1}: \Phi(\gamma))^{(1)}$ is modular but not a quasi-ideal. In general, the structure of the algebra $L / M_{L}$ is unknown.

In this section, we consider the case when $L$ has two distinct solvable modular 
maximal subalgebras. We will need the following lemma which might have some interest in itself.

Lemma 2.1. Let $F$ be any field. Let $L$ be a non-nilpotent Lie algebra and $H$ a splitting Cartan subalgebra of $L$. Let $M$ be a subalgebra of L containing $H$ such that $\operatorname{dim} L / M=1$. Let $N \triangleleft H$ such that $L^{\infty} \cap H \leq N$ and every element of $N$ is ad-nilpotent. Let $M_{1}$ denote the Fitting one-component of $M$ relative to $H$. Then, $N+M^{\infty}=N+M_{1} \triangleleft L$.

Proof. Let $L_{1}$ be the Fitting 1-component of $L$ relative to $H$. As $H$ is contained in $M$, we have that $M \cap L_{1}$ is the Fitting 1-component of $M$ relative to $H$, so that $M_{1}=L_{1} \cap M$. Since $M$ has codimension 1 in $L$, we have $\operatorname{dim} L_{1} / M_{1}=1$. We see

$$
\left[N+M_{1}, L\right]=\left[N, L_{1}\right]+[N, H]+\left[M_{1}, L_{1}\right]+\left[M_{1}, H\right] \leq\left[N, L_{1}\right]+N+\left[M_{1}, L_{1}\right]+M_{1} .
$$

Since $\operatorname{dim} L_{1} / M_{1}=1$ and since $N$ acts nilpotently on $L$, we find $\left[N, L_{1}\right] \leq M_{1}$. Now, we need prove that $\left[M_{1}, L_{1}\right] \leq M_{1}+N$. To do that, let $L=H \oplus \sum L_{\alpha}$ be the decomposition of $L$ into its root spaces relative to $H$. We have $L_{1}=\sum L_{\alpha}$ and $\sum\left[L_{\alpha}, L_{-\alpha}\right]=H \cap L^{\infty}$, see [26]. As $\operatorname{dim} L_{1} / M_{1}=1$, there exists a root $\alpha$ and a nonzero element $e \in L_{x}$ such that $L_{1}=M_{1}+F e$. We see that $L_{\beta}$ is contained in $M_{1}$ for every root $\beta \neq \alpha$. Also we see that $M_{\alpha}=L_{\alpha} \cap M$, where $M_{\alpha}$ is the root space of $M$ corresponding to $\alpha$. We find $\left[L_{\beta}, F e\right] \subseteq L_{\alpha+\beta} \subseteq M_{1}$ whenever $\beta \neq-\alpha$, and $\left[L_{-\alpha}, F e\right] \subseteq$ $\left[L_{-\alpha}, L_{\alpha}\right] \subseteq H \cap L^{\infty} \leq N$. Therefore, $\left[M_{1}, F e\right] \leq M_{1}+N$. Moreover, we have

$$
\left[M_{1}, M_{1}\right] \leq M_{1}+M^{\infty} \cap H \leq M_{1}+L^{\infty} \cap H \leq M_{1}+N .
$$

This yields $\left[M_{1}, L_{1}\right]=\left[M_{1}, M_{1}\right]+\left[M_{1}, F e\right] \leq M_{1}+N$. We conclude that $M_{1}+N$ is an ideal of $L$. Since $M^{\infty}=M_{1}+\sum\left[M_{\beta}, M_{-\beta}\right]$, we have

$$
M^{\infty}+N \leq M_{1}+\sum\left[M_{\beta}, M_{-\beta}\right]+N \leq M_{1}+L^{\infty} \cap H \leq M_{1}+N \leq M^{\infty}+N .
$$

Hence $N+M^{\infty}=N+M_{1}$. This completes the proof.

Also, we will need the following lemma.

Lemma 2.2. Let $\operatorname{char}(F)=p>3$. Let $L$ be a simple Lie algebra having a maximal subalgebra $U$ of dimension two. Then, $L \cong \mathrm{sl}(2)$ whenever $U$ is either nonabelian or modular in $L$.

Proof. First, we consider the case where $U$ is nonabelian. Let $F u$ denote the only proper ideal of $U$. Since $C_{U}(u)=F u$, we have $C_{L}(u)=F u$, since otherwise we would have $F u \triangleleft C_{L}(u) \vee U=L$, which is a contradiction. By [20, Lemma 1.3], the Engel subalgebra $E_{L}(u)$ cannot be two-dimensional nonabelian. This yields $U<E_{L}(u)$ and hence $E_{L}(u)=L$. So $u$ is a self-centralizing ad-nilpotent element of $L$. Then, we see that the subspaces of $L$ invariant under $\operatorname{ad} u$ are linearly ordered by inclusion and $u$ lies in 
a subalgebra of $L$ isomorphic to $\operatorname{sl}(2)$ (see [6, Theorem 2.8]). This yields $L \cong \operatorname{sl}(2)$. Now we assume that $U$ is modular in $L$. We prove that $U$ is nonabelian. Suppose $U$ is abelian. Then $U$ is a Cartan subalgebra of $L$. By Lemma 1.5 of [20] it follows that $\operatorname{dim} L / U$ is even. So, $\operatorname{dim} L$ is even too. Pick $x \notin U$. We have that $F x$ is not a Cartan subalgebra of $L$, since otherwise we would have that $L$ is central-simple of rank one and then, by [7], $\operatorname{dim} L$ is a power of $p$, which is a contradiction. Therefore $F x<N_{L}(F x)$. By the modularity of $U$, we have that $U \cap N_{L}(F x)$ is maximal in $N_{L}(F x)$. This yields $N_{L}(F x)=F x+N_{L}(F x) \cap U$ and hence $\operatorname{dim} N_{L}(F x)=2$. If $N_{L}(F x)$ is abelian, then $N_{L}(F x) \cap U \triangleleft U \vee N_{L}(F x)=L$, which is a contradiction. Hence $N_{L}(F x)$ is nonabelian. By the above, it follows that $L \cong \mathrm{sl}(2)$, which contradicts the fact that $U$ is abelian. We conclude that $U$ is nonabelian and hence $L \cong \mathrm{sl}(2)$. The proof is complete.

Now, we consider Lie algebras having two distinct modular, solvable maximal subalgebras.

Theorem 2.3. Let $F$ be any field. Let $L$ be a semisimple Lie algebra. Let $M$ and $K$ be distinct solvable, modular maximal subalgebras of $L$. Then the following hold:

(1) if $M \cap K=0$, then $L$ is a $\mu$-algebra;

(2) $C_{L}(x) \leq K$ for every element $x \in K, x \notin M$;

(3) if $\operatorname{char}(F) \neq 2$ and if $M$ and $K$ are strongly solvable, then either every Cartan subalgebra of $M \cap K$ is a Cartan subalgebra of $M$ and $K$ or else $M \cap K=0$;

(4) if $\operatorname{char}(F)=p>3$ and $M$ and $K$ are supersolvable and if $M \cap K$ contains a Cartan subalgebra of $L$, then $L \cong \mathrm{sl}(2)$.

Proof. We have that $M \cap K$ is a modular and maximal subalgebra of $M$ and $K$. Since $M$ and $K$ are both solvable, by [20] it follows that $\operatorname{dim} M / M \cap K=$ $\operatorname{dim} K / M \cap K=1$. Let us first suppose $M \cap K=0$. Then $\operatorname{dim} M=1$. This yields $M \cap S=0$ for every subalgebra $S$ of $L$ such that $S \not M$. Then, from the modularity of $M$ it follows that every maximal subalgebra of $L$ has dimension one. This proves (1).

In order to prove (2), let $x \in K, x \notin M$. We claim that $C_{L}(x) \cap M \leq K$. Assume not. Then there exists $y \in M, y \notin K$ such that $[x, y]=0$. We have $M=M \cap K+F y$ and $[x, M]=[x, M \cap K] \subset K$. This yields that $K+M$ is a subalgebra of $L$. Hence $L=K+M$. It follows that $\operatorname{dim} L / M=1$ and so $L=M+F x$. Let $I \subset M$ be the (unique) subspace maximal such that it is invariant under ad $x$. We have that $I$ is a solvable ideal of $L$ (see Lemma 3.7 of [6]). Since $L$ is semisimple, $I=0$. However, we have $y \in I$ since $[y, x]=0$. This contradiction proves the claim. Then we have

$$
C_{L}(x) \cap M \leq C_{L}(x) \cap M \cap K \leq C_{L}(x) \cap K .
$$

On the other hand, since $M$ is modular, we have that $C_{L}(x) \cap M$ is maximal in $C_{L}(x)$. It follows that either $C_{L}(x) \cap M=C_{L}(x) \cap K$ or $C_{L}(x) \cap K=C_{L}(x)$. In the former case, 
we get $x \in K \cap C_{L}(x) \leq M$, which is a contradiction. Therefore, $C_{L}(x) \cap K=C_{L}(x)$, so that $C_{L}(x) \leq K$.

(3): Assume $M \cap K \neq 0$ Let $C$ be a Cartan subalgebra of $M \cap K$. Suppose that $C$ is not a Cartan subalgebra of $K$. First, we prove $M \cap K \triangleleft K$. We have $C<N_{K}(C)$ and $M \cap N_{K}(C)=C$. Since $M$ is modular, $C$ is maximal and modular in $N_{K}(C)$. Since $N_{K}(C)$ is solvable, by [20] it follows that $\operatorname{dim} N_{K}(C) / C=1$. Let $K=K_{0}(C)+K_{1}(C)$ be the Fitting decomposition of $K$ relative to $C$. We have $K_{0}(C)=N_{K}(C)$ and $K_{1}(C) \subset M \cap K$. This yields

$$
[K, M \cap K]=\left[N_{K}(C)+K_{1}(C), C+K_{1}(C)\right] \leq C+K_{1}(C) \leq M \cap K .
$$

Therefore $M \cap K \triangleleft K$.

Next, we prove that every element $x \in M \cap K$ acting nilpotently on $M \cap K$ is adnilpotent on $L$. Suppose $E_{L}(x) \neq L$. As $M \cap K \triangleleft K$, we see that $x$ acts nilpotently on $K$. So $K=E_{L}(x)$. This yields $E_{M}(x)=M \cap K$. Since $\operatorname{dim} M / M \cap K=1$, it follows that the Fitting one-component $M_{1}(x)$ of $M$ relative to $\operatorname{ad}_{M} x$ has dimension one. Put $M_{1}(x)=F e$. Then, we see that $F e \triangleleft M$ and $F e \leq M^{\prime}$. So $M^{\prime}=F e+M^{\prime} \cap K$. On the other hand, since $e \notin M \cap K$ we have $M=F e+M \cap K$. We obtain $M^{\prime} \leq[F e, M \cap K]+$ $(M \cap K)^{\prime}$. This yields $(M \cap K)^{\prime}=M^{\prime} \cap K$. Since $M^{\prime} \cap K$ is maximal in $M^{\prime}$ and since $M^{\prime}$ is nilpotent, we have $M^{\prime} \cap K \triangleleft M^{\prime}$. Hence $M^{\prime} \cap K \triangleleft M$. We get $(M \cap K)^{\prime} \triangleleft M \vee K=L$. Since $L$ is semisimple, it follows that $(M \cap K)^{\prime}=0$. This yields that $M \cap K$ is abelian and so $C=M \cap K$. Since $F e \triangleleft M$, we have $[c, e]=\alpha(c) e$ where $\alpha(c) \in F$, for every $c \in C$. We see that $\alpha$ is a linear form of $C$ and a root of $L$ relative to $C$. For $1 \leq j \leq p-1$, let $S_{j \alpha}=\{v \in L \mid[c, v]=j \alpha(c) v, \forall c \in C\}$. Put $S(\alpha)=C+\Sigma_{j} S_{j \alpha}$. We see that $S(\alpha)$ is a subalgebra of $L$ containing $M$. Moreover, $S(\alpha) \neq L$ since $N_{K}(C) \neq C$. This yields $M=S(\alpha)$ and therefore $S_{j \alpha}=0$ for every $j>1$. Since $C$ is abelian, it follows that $L_{j \alpha}=0$, for every $j>1$, where $L_{j x}$ denotes the root space of $L$ corresponding to $j \alpha$ relative to $C$. This yields that $N_{L}(C) \oplus L_{\alpha}$ is a proper subalgebra of $L$ which contains $K$ and hence $L=N_{L}(C) \oplus L_{\alpha}$. We see $\left[L_{\alpha}, L_{\alpha}\right] \leq L_{2 \alpha}=0$ and $\left[L, L_{\alpha}\right] \leq L_{\alpha}$. This yields that $L_{\alpha}$ is an abelian ideal of $L$, which is a contradiction. Therefore, $E_{L}(x)=L$, as desired.

Since $M \cap K \triangleleft K$ and since $K^{\prime}$ is nilpotent, we have that every element of $K^{\prime}$ acts nilpotently on $M \cap K$. So, by the preceding paragraph, every element of $K^{\prime}$ is ad-nilpotent. Then $K^{\prime}+\operatorname{Nil}(M)$ is a nilpotent subalgebra of $M$. Since $M^{\prime}$ is nilpotent, we have $M^{\prime} \leq K^{\prime}+\operatorname{Nil}(M)$. This yields that $K^{\prime}+\operatorname{Nil}(M) \triangleleft M$ and so $K^{\prime}+\operatorname{Nil}(M) \leq \operatorname{Nil}(M)$. Thus $K^{\prime} \leq \operatorname{Nil}(M)$. Therefore, $\operatorname{Nil}(M) \cap K \triangleleft K$. On the other hand, we have that $\operatorname{Nil}(M) \cap K$ is maximal in $\operatorname{Nil}(M)$, by the modularity of $K$. Nilpotency of $\operatorname{Nil}(M)$ implies $\operatorname{Nil}(M) \cap K \triangleleft M$. Therefore, $\operatorname{Nil}(M) \cap K \triangleleft M \vee K=L$. This yields $\operatorname{Nil}(M) \cap K=0$ and hence $K$ is abelian. It follows that every element of $M \cap K$ acts trivially on $M \cap K$. By using the preceding paragraph again, we obtain that every element of $M \cap K$ is ad-nilpotent. Then, by using Engel's theorem, we obtain $M \cap K \triangleleft M$. This yields $M \cap K \triangleleft M \vee K=L$ and hence $M \cap K=0$. This contradiction completes the proof of (3).

(4): Let $C$ be a Cartan subalgebra of $L$ such that $C \leq M \cap K$. As $M$ and $K$ are both supersolvable, we see that ad $c$ is split for every $c \in C$. Put $T=M \cap K$. We have 
$\operatorname{dim} M / T=\operatorname{dim} K / T=1$, since $M$ and $K$ are both modular. Thus there exists only one root $\alpha$ of $C$ for which the corresponding root space $M_{\alpha}$ of $M$ is not contained in $T$. Also, we see that there exists only one root $\beta$ of $C$ for which the corresponding root space $K_{\beta}$ of $K$ is not contained in $T$. Pick $e \in M_{\alpha}, e \notin T$ and $f \in K_{\beta}, f \notin T$. Assume $[e, f] \in M+K$. This yields that $M+K$ is a subalgebra of $L$ and hence $L=M+K$. It follows $\operatorname{dim} L / M=1=\operatorname{dim} L / K$. Since $M$ is solvable and $L$ is semisimple, we see that $M$ contains no nonzero ideals of $L$. It follows that $L \cong \operatorname{sl}(2)$ (see [2, Theorems 3.1 and 3.2]). Suppose then that $[e, f] \notin M+K$.

Now we claim that $M^{\prime} \cap C$ acts nilpotently on $K$. If $M^{\prime} \cap C=0$ there is nothing to do. Assume $M^{\prime} \cap C \neq 0$. Since $M^{\prime}$ is nilpotent, $M^{\prime} \cap C$ acts nilpotently on each root space of $M$ relative to $C$. So we only need prove that $\beta\left(M^{\prime} \cap C\right)=0$. Assume $\beta\left(M^{\prime} \cap C\right) \neq 0$. Then, $(\alpha+\beta)\left(M^{\prime} \cap C\right) \neq 0$. Put $L(\alpha+\beta)=C \oplus L_{\alpha+\beta} \oplus \cdots \oplus L_{(p-1)(\alpha+\beta)}$. Since $[e, f] \neq 0$, we have $L_{\alpha+\beta} \neq 0$. We see that $L(\alpha+\beta)$ is a proper subalgebra of $L$ and $L(\alpha+\beta) \cap K=C$. Since $K$ is modular, $C$ is maximal in $L(\alpha+\beta)$. Since $C^{\prime} \leq M^{\prime} \cap K^{\prime}$ and since $M^{\prime}$ and $K^{\prime}$ are both nilpotent, we see that $C^{\prime}$ acts nilpotently on $M \vee K=L$. This yields that the Lie algebra $\{\operatorname{ad} c \mid c \in C\}$ of linear transformations of $L$ is triangulable on $F$ (see [26]). So, there exists $0 \neq g \in L_{(\alpha+\beta)}$ such that $[c, g]=(\alpha+\beta)(c) g$ for every $c \in C$. Then, we have $C<C+F g \leq L_{(\alpha+\beta)}$. This yields $L_{2(\alpha+\beta)}=0$ and therefore $[[e,[e, f]], f] \in L_{2(x+\beta)}=0$. By (2), it follows that $[e,[e, f]] \in C_{L}(f) \leq K$. Since $(2 \alpha+\beta)\left(M^{\prime} \cap C\right) \neq 0$, we see that $K \cap L_{2 \alpha+\beta}=0$. So, $[e,[e, f]]=0$. By (2) again, it follows that $[e, f] \in M$, which is a contradiction. The proof of the claim is complete. Analogously, we obtain that $K^{\prime} \cap C$ acts nilpotently on $M$.

By the above claim, it follows that $M^{\prime} \cap C+K^{\prime} \cap C$ acts nilpotently on $M$ and $K$. By Lemma 2.1, it follows that $M^{\prime} \cap C+K^{\prime} \cap C+T_{1}(C)$ is an ideal of $M$ and $K$. Since $L$ is semisimple, $M^{\prime} \cap C+K^{\prime} \cap C+T_{1}(C)=0$. We deduce that $T_{1}(C)=0$. So $T=C, C$ is abelian, $\alpha$ is the only root of $C$ in $M$ and $\beta$ is the only root of $C$ in $K$. Now let us suppose $\alpha \neq j \beta$ for every $1 \leq j \leq p-1$. We see $\alpha \neq j(\alpha+\beta)$, for every $1 \leq j \leq p-1$. So, $L(\alpha+\beta) \neq L$ and $L(\alpha+\beta) \cap M=C$. Since $M$ is modular, $C$ is maximal in $L(\alpha+\beta)$ ). As above, this yields $L_{2(\alpha+\beta)}=0$ and we get a contradiction. Therefore $\alpha=j \beta$ for some $1 \leq j \leq p-1$. Pick $c \in \operatorname{ker} \alpha$. We have $[M, c]=[K, c]=0$. So, $c \in Z(L)=0$. Therefore $\operatorname{ker} \alpha=0$. As $C$ is abelian, $\alpha$ is a linear form of $C$. Thus $\operatorname{dim} C=1$. This yields that $M$ and $K$ are both nonabelian of dimension two. Assume that $L$ is not simple. Then, take a minimal ideal $N$ of $L$. We have that $M \cap N$ is maximal and modular in $N$. This yields that every maximal subalgebra of $N$ has dimension one and so $N$ is a $\mu$-algebra. Since $M \cap N \triangleleft M$ and $K \cap N \triangleleft K$, we see $M \cap N=F e \leq N_{\alpha}$ and $K \cap N=F f \leq N_{\beta}$. Moreover we have $\left[N_{\beta}, N_{(p-1) \beta}\right] \leq N \cap C=0$. This yields $N_{(p-1) \beta}=0$, since $N$ is a $\mu$ algebra. From this it follows that $N_{j \beta}=0$, for every $1<j \leq p-1$. As $N_{\alpha} \neq 0$, we get $\alpha=\beta$. But then we have $[e, f] \in\left[N_{\beta}, N_{\beta}\right] \leq N_{2 \beta}=0$, which is a contradiction. Therefore $L$ is simple. Then, by Lemma 2.2 it follows that $L \cong \operatorname{sl}(2)$. The proof is now complete.

We finish this section by considering Lie algebras having two distinct modular maximal subalgebras in the case where one of them is isomorphic to sl(2). 
Lemma 2.4. Let $\operatorname{char}(F)>3$. Let $M$ and $K$ be distinct modular maximal subalgebras of $L$. Assume $M \cong \operatorname{sl}(2)$. Then $M \triangleleft L$.

Proof. Assume that $M$ is not an ideal of $L$. Let $0 \neq N \triangleleft L$. We see $M \cap N=0$. So, $\operatorname{dim} N=1$ by the modularity of $M$. Since $L=M+N$, we have $L / N \cong \operatorname{sl}(2)$. Then we have $L=N \oplus L^{\prime}$ (see [20, Lemma 4.1]). This yields $N=Z(L)$ and hence $M \triangleleft L$, which is a contradiction. Therefore $L$ is simple. By the modularity of $K$, we have that $M \cap K$ is modular and maximal in $M$. This yields $\operatorname{dim} M \cap K=2$. Then there exists a basis $e, h$ for $M \cap K$ with product $[h, e]=e$. We see $M \leq E_{L}(e)$. If $E_{L}(e)=M$, then we have $C_{L}(e)=C_{M}(e)=F e$. But then, as $\operatorname{char}(F)>3$, Lemma 1.3 of [20] applies and $E_{L}(e)$ is not isomorphic to $\mathrm{sl}(2)$. This contradiction shows that $E_{L}(e)=L$. This yields $C_{L}(e) \neq F e$, since otherwise the subspaces of $L$ invariant under ad $e$ would be linearly ordered by inclusion and so $M=K$, which is a contradiction. We have $C_{L}(e) \cap M=F e$. Modularity of $M$ implies that $F e$ is maximal in $C_{L}(e)$. This yields that $C_{L}(e)$ is abelian of dimension two. It follows that $\operatorname{ker}\left(\operatorname{ad}^{2} e\right)$ is a subalgebra of $L$. Since $M \cap \operatorname{ker}\left(\operatorname{ad}^{2} e\right)=F e+F h$, from the modularity of $M$ it follows that $\operatorname{dim}\left(\operatorname{ker}^{2} \operatorname{ad}^{2} e\right)=3$. So $\operatorname{ker}\left(\operatorname{ad}^{2} e\right)=F h+C_{L}(e)$. On the other hand, since 1 is an eigenvalue of $\operatorname{ad}_{M} h$, we have that -1 is also an eigenvalue of $\operatorname{ad}_{M} h$. So, there exists $f \in M$ such that $[h, f]=-f$. We have $[e, f]=h$ and so $\left[e,\left[e,\left[f, C_{L}(e)\right]\right]\right]=\left[h, C_{L}(e)\right] \leq \operatorname{ker}\left(\operatorname{ad}^{2} e\right)$. This yields that $M+\operatorname{ker}\left(\operatorname{ad}^{2} e\right)$ is a subalgebra of $L$ and hence $L=M+\operatorname{ker}\left(\operatorname{ad}^{2} e\right)$. We get $\operatorname{dim} L / M=1$. But then, by using [1] we obtain that either $M \triangleleft L$ or $M$ is solvable, which is a contradiction. The proof is complete.

\section{On $M(1)$-algebras}

A Lie algebra $L$ is said to be an $M(1)$-algebra if all of its maximal subalgebras are modular in $L$. Clearly, the class of $M(1)$-algebras is closed under homomorphic images. For fields of characteristic zero, in [17] it is proved that if $L$ is $M(1)$ then $L / \operatorname{Fr}(L)$ is lower semimodular. We recall that for such fields the Frattini subalgebra of any Lie algebra $L$ is a characteristic ideal of $L$ (see [14]). This fails in the prime characteristic case, although the only known examples of Lie algebras in which the Frattini subalgebra is not an ideal are the three-dimensional split simple algebras over a field of characteristic two and the simple Lie algebra of Cartan type $\mathcal{H}(2: \underline{1}: \Phi(\gamma))^{(1)}$, see [21].

In this section, we obtain some results on $M(1)$-algebras which will be used later. First we give the following two easy lemmas.

Lemma 3.1. Let $F$ be any field and let $L$ be a Lie algebra. Then the following hold:

(1) $Z\left(L^{(\infty)}\right) \leq \phi(L)$.

(2) If $N \triangleleft L$ and if $\phi(N) \triangleleft L$, then $\phi(N) \leq \phi(L)$.

(3) Each maximal subalgebra of $L$ contains either $R(L)$ or $L^{(\infty)}$ if and only if $R(L) \cap L^{(\infty)} \leq \phi(L)$ 
Proof. Let $M$ be a maximal subalgebra of $L$. To prove (1), assume $Z\left(L^{(\infty)}\right) \leq M$. Then we have $L=M+Z\left(L^{(\infty)}\right)$. This yields $L^{(\infty)}=Z\left(L^{(\infty)}\right)+\left(M \cap L^{(\infty)}\right)$ and hence $M \cap Z\left(L^{(\infty)}\right) \triangleleft L^{(\infty)}$. We see that $L^{(\infty)} /\left(M \cap L^{(\infty)}\right)$ is a homomorphic image of $Z\left(L^{(\infty)}\right)$ and so it is abelian. This yields $\left(L^{(\infty)}\right)^{(\infty)} \leq M \cap L^{(\infty)}<L^{(\infty)}$, which is a contradiction. Therefore $Z\left(L^{(\infty)}\right) \leq M$. To prove (2), assume that $\phi(N) \notin M$. As $\phi(N) \triangleleft L$, we have that $\phi(N)+M$ is a subalgebra of $L$ and so $\phi(N)+M=L$. This yields $N=\phi(N)+$ $(M \cap N)$ and therefore $N=M \cap N$, which is a contradiction. (3): Let $R(L) \cap L^{(\infty)} \leq \phi(L)$. Assume that $R(L) \not M$. Then we have $L=R(L)+M$ and $M^{(\infty)}+R(L) \triangleleft L$. Also, we see that $L /\left(M^{(\infty)}+R(L)\right)$ is solvable. Since $M^{(\infty)} \leq L^{(\infty)}$, it follows that $R(L)+L^{(\infty)}=$ $R(L)+M^{(\infty)}$. This yields $M^{(\infty)}=L^{(\infty)}$ and hence $L^{(\infty)} \leq M$. This completes the proof of (3) in one direction. The converse is clear.

Lemma 3.2. Let $F$ be any field. For a Lie algebra $L$, the following are equivalent:

(i) L is an M(1)-algebra;

(ii) for each maximal subalgebra $M$ of $L, M \cap K$ is modular and maximal in $K$ for every maximal subalgebra $K$ of $L$ different from $M$.

Proof. (ii) $\Rightarrow$ (i): Let $M$ be a maximal subalgebra of $L$. By Lemma 1 of [17], we need only prove that $M \cap S$ is maximal in $S$, for every proper subalgebra $S$ of $L$ such that $S \leq M$. Take a maximal subalgebra $K$ of $L$ containing $S$. Then we have that $M \cap K$ is a modular maximal subalgebra of $K$. This yields that $M \cap S$ is maximal in $S$. (i) $\Rightarrow$ (ii) is clear.

Our main results on arbitrary $M(1)$-algebras are the two following propositions which will be used in the sequel.

Proposition 3.3. Let $F$ be any field. Let $L$ be an $M(1)$-algebra. Then the following hold:

(1) $R(L) \cap L^{u} \leq \phi(L)$.

(2) $R(L)$ is supersolvable.

(3) If $L^{u}=L$, then $R(L)=\phi(L)$.

Proof. (1): Let $M$ be a maximal subalgebra of $L$. Assume that $R(L) \leq M$. Then by the modularity of $M, R(L) \cap M$ is modular and maximal in $R(L)$. By [20], it follows that $\operatorname{dim} R(L) /(M \cap R(L))=1$. Since $L=R(L)+M$, we have $\operatorname{dim} L / M=1$. Let $M_{L}$ denote the largest ideal of $L$ contained in $M$. By [2, Theorems 3.1 and 3.2], we have either $\operatorname{dim} L / M_{L} \leq 2$ or $L / M_{L}$ is semisimple. In the latter case, since $R(L)+M_{L} / M_{L}$ is a solvable ideal of $L / M_{L}$, we have $R(L)+M_{L}=M_{L}$. This yields $R(L) \leq M_{L} \leq M$, which is a contradiction. Therefore, $\operatorname{dim} L / M_{L}=2$. We have $L^{u} \leq M_{L} \leq M$. Then, (1) follows from Lemma 3.1(3). (2): Since $\phi(L) \leq R(L)$ (see [14]), by (1) it follows that $R(L) / \phi(L)$ is a homomorphic image of $R(L)+L^{u} / L^{u}$. So, $R(L) / \phi(L)$ is supersolvable. 
Then by [4] it follows that $R(L)$ is supersolvable too. (3) follows from (1) and from the fact that $\phi(L)$ is a nilpotent ideal of $L$ (see [14]).

Proposition 3.4. Let $L$ be an M(1)-algebra over any field. Assume that $\operatorname{Fr}(L)=M \cap K$ for some maximal subalgebras $M$ and $K$ of $L$. Then the following hold:

(i) $\operatorname{Fr}(L)$ is a modular and maximal subalgebra in each maximal subalgebra of $L$;

(ii) $\operatorname{Fr}(L)$ is modular in $L$.

Proof. (i): From the modularity of $M$ and $K$ it follows that $\operatorname{Fr}(L)$ is modular and maximal in $M$ and $K$. Now let $T$ be any maximal subalgebra of $L$ different from $M$. We have $\operatorname{Fr}(L) \leq M \cap T<M$ and so $\operatorname{Fr}(L)=M \cap T$. By the modularity of $M$ again, it follows that $\operatorname{Fr}(L)$ is modular and maximal in $T$. (ii): Let $S$ be a proper subalgebra of $L$ such that $S \not z \operatorname{Fr}(L)$. We have $\operatorname{Fr}(L) \vee S \neq L$. Then, by using (i) we obtain that $\operatorname{Fr}(L) \vee S$ is maximal in $L$ and $\operatorname{Fr}(L)$ is modular and maximal in $\operatorname{Fr}(L) \vee S$. This yields that $\operatorname{Fr}(L) \cap S$ is maximal in $S$. Therefore, the mapping $\phi_{\operatorname{Fr}(L) . S}:[\operatorname{Fr}(L): \operatorname{Fr}(L) \vee S] \ni U \rightarrow U \cap S \in[\operatorname{Fr}(L) \cap S: S]$ is a bijection. So $\operatorname{Fr}(L)$ is modular in $L$, as required.

\section{4. $s \chi$-algebras}

A Lie algebra $L$ is said to be a $\chi$-algebra if every maximal subalgebra of $L$ has codimension one in $L$. A Lie algebra $L$ is said to be an s $\chi$-algebra if every subalgebra of $L$ (including $L$ itself) is a $\chi$-algebra. The aim of this section is to determine the structure of an $s \chi$-algebra over any field $F$ with $\operatorname{char}(F) \neq 2$. For such fields, a Lie algebra $L$ such that $\phi(L)=0$ is a $\chi$-algebra if and only if either $L$ is supersolvable or else $\sqrt{F} \leq F$ and $L \cong U \oplus S$ where $U$ is supersolvable and $S \cong \operatorname{sl}(2)$, according to Towers [16]. For $s \chi$-algebras we have the following.

Theorem 4.1. Let $\operatorname{char}(F) \neq 2$. (i) If $\sqrt{F} \leq F$, then the supersolvable algebras are the only s $\chi$-algebras.

(ii) If $\sqrt{F} \leq F$, then a Lie algebra $L$ is an s $\chi$-algebra if and only if either $L$ is supersolvable or $L / R(L) \cong \mathrm{sl}(2) \cong L^{u} / \phi\left(L^{u}\right)$ and every strongly solvable subalgebra of $L$ is supersolvable.

Proof. (i): Let $\sqrt{F} \succeq F$ and let $L \in \chi$. By Towers [16], we have that $L / \phi(L)$ is supersolvable. Then, by [4], it follows that $L$ is supersolvable. (ii): Let $\sqrt{F} \leq F$ and let $L \in \mathrm{s} \chi$. By using Towers [16] we obtain that $L / R(L) \cong \operatorname{sl}(2), L=R(L)+L^{u}$ and $R(L) \cap L^{u} \leq \phi(L)$. This yields that $R(L) / \phi(L)$ is supersolvable and hence $R(L)$ is also supersolvable by [4]. Also, we see that $L=R(L)+L^{(\infty)}$. So, $L^{(\infty)}=L^{u}$. We now have $\left(L^{u}\right)^{u}=L^{u}$. Then, by using Proposition $3.3(3)$, we obtain that $R\left(L^{u}\right)=\phi\left(L^{u}\right)$. This completes the proof of (ii) in one direction. To prove the converse, let $L$ be a counterexample of minimal dimension. As $L / R(L) \cong \mathrm{sl}(2) \cong L^{u} / \phi\left(L^{u}\right)$, we have 
$\phi\left(L^{u}\right)=R\left(L^{u}\right)=R(L) \cap L^{u}$. So, $\phi\left(L^{u}\right) \triangleleft L$. By Lemma 3.1(2) it follows that $\phi\left(L^{u}\right) \leq \phi(L)$. Then, by Towers [16], we have $L \in \chi$. As $L \notin \mathrm{s} \chi$, there exists $S<L$ such that $S \notin \chi$. Take a maximal subalgebra $M$ of $L$ containing $S$. We see that $M \notin s \chi$. Also, we have that either $M \geq R(L)$ or $M \geq L^{(\infty)}$ by Lemma 3.1(3). Let us first assume that $R(L) \leq M$. Then, we have $M=R(L)+M \cap L^{u}$. So, $M^{\prime} \leq R(L)^{\prime}+\left(M \cap L^{u}\right)^{\prime}+\left[R(L), L^{u}\right]$. Since $R(L) / \phi(L)$ is supersolvable, so is $R(L)$ (see [4]). Thus $R(L)^{\prime}$ is nilpotent. Since $L^{u} / \phi\left(L^{u}\right) \cong \mathrm{sl}(2)$, we have that $\left(M \cap L^{u}\right)^{\prime}$ is nilpotent too (see the proof of Corollary 2.9 of [23]). Since $\left[R(L), L^{u}\right] \leq R(L) \cap L^{u} \leq \phi\left(L^{u}\right)$ and since $\phi\left(L^{u}\right)$ is a nilpotent ideal of $L^{u}$, it follows that $\left[R(L), L^{u}\right]$ is also nilpotent. We deduce that $M^{\prime}$ is nilpotent. Then, by our hypothesis, $M$ is supersolvable. So $M \in \mathrm{s} \chi$, which is a contradiction. Therefore $L^{(\infty)} \leq M$. Since $R(L)$ is supersolvable and since $L=R(L)+L^{(\infty)}$, we see that $L^{(\infty)}=L^{4}$. This yields $M^{u}=L^{u}$. We conclude that $M / R(M) \cong s l(2) \cong M^{u} / \phi\left(M^{u}\right)$. Then, by the minimality of $L$ it follows that $M \in \mathrm{s} \chi$. This contradiction completes the proof.

Corollary 4.2. Let $F$ be algebraically closed and $\operatorname{char}(F) \neq 2$. For a nonsolvable Lie algebra $L$, the following are equivalent:

(i) $L \in \mathrm{s} \chi$,

(ii) $L$ and $L^{u}$ are $\chi$-algebras,

(iii) $L / R(L) \cong \mathrm{sl}(2) \cong L^{u} / \phi\left(L^{u}\right)$.

In the case where $F$ is algebraically closed, we have that the $s \chi$-algebras $L$ such that $L^{\prime}=L$ are precisely the non-solvable, minimal non-supersolvable Lie algebras.

\section{Lower semimodular Lie algebras}

The aim of this section is to study the structure of a lower semimodular Lie algebra over a field $F$ of characteristic greater than three. Clearly, the class of lower semimodular Lie algebras is closed under subalgebras and homomorphic images. It is well-known that a solvable Lie algebra is lower semimodular if and only if it is supersolvable. It is easy to see that the algebra sl(2) is lower semimodular if and only if $\sqrt{F} \leq F$. Clearly, every $\mu$-algebra is lower semimodular.

Our first result is that the algebra $\mathrm{sl}(2)$ and the $\mu$-algebras are the only semisimple lower semimodular Lie algebras having more than one solvable maximal subalgebra.

Proposition 5.1. Let $\operatorname{char}(F)>3$. Let $L$ be a semisimple lower semimodular Lie algebra. Then, the following hold:

(i) every Cartan subalgebra $C$ of a solvable maximal subalgebra $U$ of $L$ is also a Cartan subalgebra of $L$;

(ii) if $L$ has more than one solvable maximal subalgebra, then either $L \cong \operatorname{sl}(2)$ or $L$ is a $\mu$-algebra. 
Proof. (i): Assume that $C$ is not a Cartan subalgebra of $L$. We see that $C<L_{0}<L$, where $L_{0}$ denotes the Fitting null-component of $L$ relative to $C$. Take a maximal subalgebra $M$ of $L$ containing $L_{0}$. We see that $C$ is not a Cartan subalgebra of $M$. Then, by Theorem 2.3(3) it follows that $M$ is nonsolvable. Since $M \cap U$ is a solvable maximal subalgebra of $M$, we have $R(M)<M \cap U$. Also, we have that $C+R(M) / R(M)$ is a Cartan subalgebra of $M \cap U / R(M)$. Then, arguing by induction on $\operatorname{dim} L$, we obtain that $C+R(M) / R(M)$ is a Cartan subalgebra of $M / R(M)$. But then, by [4], it follows that $C$ is a Cartan subalgebra of $M$, which is a contradiction.

(ii): Let $U$ and $U^{*}$ be distinct solvable maximal subalgebras of $L$. If $U \cap U^{*}=0$, then by Theorem 2.3(1) it follows that $L$ is a $\mu$-algebra. Assume then $U \cap U^{*} \neq 0$. Take a Cartan subalgebra $C$ of $U \cap U^{*}$. By Theorem 2.3(3), we have that $C$ is a Cartan subalgebra of $U$. By (i) it follows that $C$ is a Cartan subalgebra of $L$. Then, by Theorem 2.3(4) we have that $L \cong \operatorname{sl}(2)$. This completes the proof.

Next, we study lower semimodular Lie algebras which are not in the class $s \chi$.

Theorem 5.2. Let $\operatorname{char}(F)>3$. Let $L$ be a lower semimodular Lie algebra such that $L \notin \mathrm{s} \chi$. Then there exists $S \leq L$ such that $S / R(S)$ is a $\mu$-algebra.

Proof. Let $L$ be a counterexample of minimal dimension. Then we see that every proper subalgebra of $L$ is in the class $s \chi$. Let $R(L) \neq 0$. Then, by the minimality of $L$ we have $L / R(L) \in \mathrm{s} \chi$. So, $L / R(L) \cong \operatorname{sl}(2)$ and $\sqrt{F} \leq F$ by Theorem 4.1 . This yields $L=R(L)+L^{u}$ and hence $R\left(L^{u}\right)=R(L) \cap L^{u}$ and $L^{u} / R\left(L^{u}\right) \cong \operatorname{sl}(2)$. On the other hand, we have $R\left(L^{u}\right)=\phi\left(L^{u}\right)$ by Proposition 3.3. Then by Theorem 4.1 it follows that $L \in \mathrm{s} \chi$, which is a contradiction. Therefore $R(L)=0$. Then, by using Proposition 5.1 we obtain that $L$ has at most one solvable maximal subalgebra. So there must exist at least two nonsolvable maximal subalgebras $S$ and $S^{*}$ of $L$. By Theorem 4.1 we have $S / R(S) \cong \operatorname{sl}(2)$ and $\sqrt{F} \leq F$. By Lemma 2.4, we have $R(S) \neq 0$. We claim that $R(S) \leq \operatorname{Fr}(L)$. Assume not. Then, there exists a maximal subalgebra $M$ of $L$ such that $R(S) \leq M$. Since $M \cap S$ is maximal in $S$, we have $S^{(\infty)} \leq M$ by Proposition 3.3 and Lemma 3.1. This yields $(M \cap S)^{(\infty)}=S^{(\infty)}$ and $M \cap S$ is nonsolvable. Thus $M$ is nonsolvable and so $M / R(M) \cong \operatorname{sl}(2)$. It follows that $R(M) \not M M S$. Since $M \cap S$ is maximal in $M$, by Proposition 3.3 and Lemma 3.1 again we have $M^{(\infty)} \leq S$. This yields $M^{(\infty)}=(M \cap S)^{(\infty)}$ and hence $M^{(\infty)}=S^{(\infty)}$. It follows that $S^{(\infty)} \triangleleft M \vee S=L$. Since $S / R(S)$ is simple, we see that $R\left(S^{(\infty)}\right)=R(S) \cap S^{(\infty)} \triangleleft S$. Then we get $R\left(S^{(\infty)}\right)=R\left(M^{(\infty)}\right) \triangleleft S \vee M=L$. As $L$ is semisimple, $R\left(S^{(\infty)}\right)=0$. Therefore $S^{(\infty)}$ is an ideal of $L$ isomorphic to $\operatorname{sl}(2)$. Since every derivation of $\operatorname{sl}(2)$ is inner, it follows that $L=S^{(\infty)} \oplus C_{L}\left(S^{(\infty)}\right)$. We see that $C_{L}\left(S^{(\infty)}\right)$ is semisimple too. So $C_{L}\left(S^{(\infty)}\right) \cong \mathrm{sl}(2)$, by the minimality of $L$. We deduce that $L$ is isomorphic to a direct sum of two copies of $\operatorname{sl}(2)$. But then we see that the diagonal subalgebra of $L$ is not modular in $L$, which is a contradiction. Therefore $R(S) \leq \operatorname{Fr}(L)$, as claimed. We conclude that $R(S)+R\left(S^{*}\right) \leq$ $\operatorname{Fr}(L) \leq S \cap S^{*}$. Since $S \cap S^{*}$ is maximal in $S$, we have $\operatorname{dim} S \cap S^{*} / R(S)=2$. This yields that either $S \cap S^{*}=\operatorname{Fr}(L)$ or $R(S)<R(S)+R\left(S^{*}\right)=\operatorname{Fr}(L) \triangleleft S \cap S^{*}$. In the latter case, we have that there exists a maximal subalgebra $M$ of $L$ such that $S \cap S^{*} \not M$ and so 
$S \cap S^{*} \neq M \cap S$. It follows that $\operatorname{Fr}(L) / R(S)$ is contained in two distinct maximal subalgebras of $S / R(S)$ and it is an ideal in one of them, which contradicts the fact that $S / R(S) \cong \mathrm{sl}(2)$. Therefore $S \cap S^{*}=\operatorname{Fr}(L)$. Then, by using Proposition 3.4(i) we obtain that $\operatorname{Fr}(L)$ is a maximal subalgebra of $\operatorname{Fr}(L) \vee F x$, for every $x \in L, x \notin \operatorname{Fr}(L)$. Since $\operatorname{Fr}(L) \vee F x \neq L$, we see $\operatorname{Fr}(L) \vee F x \in \chi$. So, $\operatorname{dim}[\operatorname{Fr}(L) \vee F x / \operatorname{Fr}(L)]=1$. Hence $[\operatorname{Fr}(L), F x] \leq \operatorname{Fr}(L)+F x$, for every $x \in L$. So $\operatorname{Fr}(L)$ is a quasi-ideal of $L$. By Amayo [1], it follows that $\operatorname{Fr}(L) \triangleleft L$. But then we have that $\operatorname{Fr}(L)$ is a nilpotent ideal of $L$ (see [14]). This contradiction completes the proof.

Corollary 5.3. Let $\operatorname{char}(F)>3$. Assume that there are no $\mu$-algebras over $F$. Then, every lower semimodular Lie algebra is an $\mathrm{s} \chi$-algebra.

Corollary 5.4. Let $F$ be algebraically closed with $\operatorname{char}(F)>3$. Then, a Lie algebra $L$ is lower semimodular if and only if either $L$ is supersolvable or $L / R(L) \cong L^{(\infty)} / \phi\left(L^{(\infty)}\right) \cong \mathrm{sl}(2)$.

We say that a Lie algebra $L$ is completely split if ad $x$ is split for every $x \in L$. Next, we determine the structure of lower semimodular Lie algebras $L$ in the case where $L / R(L)$ is isomorphic to a direct sum of simple Lie algebras which are not completely split. To do that, we need the following lemma which might have some interest in itself.

Lemma 5.5. Let $L$ be a Lie algebra and let $N \triangleleft L$ be such that $L / N$ is isomorphic to a direct sum of simple Lie algebras which are not completely split. Assume that $\left.(\mathrm{ad} x)\right|_{N}$ is split for every $x \in L, x \notin N$. Then $L=N+C_{L}(N)$.

Proof. Let $P / N$ be a simple ideal of $L / N$. By our hypothesis, there exists $x \in P, x \notin N$ such that ad $x$ is not split on $P / N$. Let $S_{P}(x)$ denote the largest subspace of $P$ on which ad $x$ is split. It is well-known that $S_{P}(x)$ is a subalgebra of $P$ and that there exists a subspace $K_{P}(x)$ of $P$ invariant under ad $x$ and such that $L=S_{P}(x) \oplus K_{P}(x)$, see [4]. It is easy to see that $\left[S_{P}(x), K_{P}(x)\right] \leq K_{P}(x)$. We have $N \leq S_{P}(x)<P$. It follows that $\left[N, K_{P}(x)\right] \leq N \cap\left[S_{P}(x), K_{P}(x)\right] \leq N \cap K_{P}(x) \leq S_{P}(x) \cap K_{P}(x)=0$. So $K_{P}(x) \subseteq$ $C_{P}(N)$. This yields $C_{P}(N) \leq N$. Since $P / N$ is simple, $P=N+C_{P}(N)$. Therefore $L=N+C_{L}(N)$.

Proposition 5.6. Let $L$ be a Lie algebra such that every solvable subalgebra is supersolvable. Assume that $L / R(L)$ is isomorphic to a direct sum of simple Lie algebras which are not completely split. Then $\left[R(L), L^{(\infty)}\right]=0$.

Proof. Pick $x \in L, x \notin R(L)$. We have that $R(L)+F x$ is supersolvable. This yields that $\left.(\operatorname{ad}(x))\right|_{R(L)}$ is split. Then, Lemma 2.5 applies and $L=R(L)+C_{L}(R(L))$. This yields $L^{(\infty)} \leq C_{L}(R(L))$

Our next task is to prove that every semisimple lower semimodular Lie algebra can be decomposed into a direct sum of simple ideals. First, we prove that every 
supersimple ideal of a lower semimodular Lie algebra $L$ is a direct summand of $L$. We will use the fact that for each nonzero element $a$ of a supersimple Lie algebra $L$ there exists an element $b \in L$ such that $L=F a \vee F b$ and that there are only finitely many maximal subalgebras of $L$ containing $a$, provided $\operatorname{char}(F)>3$ (see the proof of Theorem 4 of [24]).

Lemma 5.7. Let $\operatorname{char}(F)>3$. Let $L$ be a lower semimodular Lie algebra. Let $N$ be an ideal of $L$ which is supersimple. Then $L=N \oplus C_{L}(N)$.

Proof. Let $L$ be a counterexample of minimal dimension. We see that $\operatorname{dim} L / N=1$ and $N$ is the unique proper ideal of $L$. Let $M \neq N$ be a maximal subalgebra of $L$. We have that $M \cap N$ is a modular maximal subalgebra of $N$. Assume $N$ is a $\mu$-algebra. Then $\operatorname{dim} M \cap N=1$ and $\operatorname{so} \operatorname{dim} M=2$. By Proposition 5.1(ii) it follows that $L$ is simple, a contradiction. Therefore $N$ is not a $\mu$-algebra. This yields $\operatorname{dim} M \cap N>1$ and hence $M \cap N$ is a supersimple ideal of $M$. By the minimality of $L$, it follows that $M=(M \cap N) \oplus Z(M)$. We see that every maximal subalgebra of $M$ different from $M \cap N$ must contain $Z(M)$ (see Lemma 3.1). Now we take a third maximal subalgebra $M^{*}$ of $L$. We have that $M \cap M^{*}$ is maximal in $M$. If $M \cap M^{*}=M \cap N$, then $M \cap N=M^{*} \cap N$. So, $M \cap N \triangleleft M \vee M^{*}=L$, which contradicts the simplicity of $N$. Therefore $M \cap M^{*} \geq Z(M)$. Similarly, we obtain $M \cap M^{*} \geq Z\left(M^{*}\right)$. We deduce that $Z(M)+Z\left(M^{*}\right)$ is contained in every maximal subalgebra of $L$ different from $N$. Therefore $\left(Z(M)+Z\left(M^{*}\right)\right) \cap N$ is contained in the Frattini subalgebra of $L$. We see that $Z(M) \neq Z\left(M^{*}\right)$, since otherwise we would have $Z(M) \triangleleft M \vee M^{*}=L$, which is a contradiction. This yields $N \cap\left(Z(M)+Z\left(M^{*}\right)\right) \neq 0$. Pick $0 \neq a \in N \cap\left(Z(M)+Z\left(M^{*}\right)\right)$. We see that $F a \leq \operatorname{Fr}(L)$. By Theorem 4 of [24], there exists $b \in N$ such that $N=F a \vee F b$. Let $0 \neq \lambda \in F$. Since every proper subalgebra of $L$ containing an Engel subalgebra of $L$ must be self-normalizing (see [4]) and since $L \neq E_{L}(a+\lambda b)$, there exists a maximal subalgebra $M(\lambda)$ of $L$ different from $N$ containing $a+\lambda b$. Now, let $0 \neq \lambda^{\prime} \in F, \lambda^{\prime} \neq \lambda$. Assume $M(\lambda) \cap N=M\left(\lambda^{\prime}\right) \cap N$. Then, $a+\lambda b$ and $a+\lambda^{\prime} b$ lie in $M(\lambda)$. So $a, b \in M(\lambda) \cap N$. This yields $N \leq M(\lambda)$, which is a contradiction. On the other hand, as $N$ is supersimple, we have that the ground field $F$ must be infinite (see [24]). Therefore, we conclude that there are infinitely many maximal subalgebras of $N$ containing Fa. However, in the proof of Theorem 4 of [24] it is proved that this cannot occur in the case when the ground field has characteristic greater than three. The proof is now complete.

We will say that two supersimple Lie algebras $P_{1}$ and $P_{2}$ are strongly non-isomorphic if none of the subalgebras of $P_{1}$ of dimension greater than one is isomorphic to a subalgebra of $P_{2}$.

Lemma 5.8. Let $L=P_{1} \oplus \cdots \oplus P_{r}$, where the $P_{i}$ are supersimple ideals of $L$ and mutually non-isomorphic. Let $M$ be a maximal subalgebra of $L$. Then the following hold: 
(1) $M=\left(M \cap P_{i}\right) \oplus P_{1} \oplus \cdots \oplus \hat{P}_{i} \oplus \cdots \oplus P_{r}$ for some $1 \leq i \leq r$ (where $\hat{P}_{i}$ indicates that $P_{i}$ is omitted from the direct sum).

(2) Either $M$ is isomorphic to a direct sum of supersimple Lie algebras or else $\operatorname{dim} R(M)=1$ and $M^{(\infty)} \triangleleft L$.

(3) $M$ contains at least one solvable subalgebra of $L$ of maximal dimension.

(4) Every solvable subalgebra of $L$ of maximal dimension has dimension $r$.

(5) $L$ is lower semimodular if and only if $P_{1}, \ldots, P_{r}$ are lower semimodular and pairwise strongly non-isomorphic.

Proof. (1)-(4) are straightforward. (5) follows from (1) and Lemma 3.2.

Now we can prove the following.

Theorem 5.9. Let $\sqrt{F} \notin F$ and $\operatorname{char}(F)>3$. Then every semisimple lower semimodular Lie algebra is isomorphic to a direct sum of supersimple Lie algebras.

Proof. Let $L$ be a counterexample of minimal dimension. First, we claim that $L$ is simple. Let $N$ be a proper minimal ideal of $L$. Then we have $N^{(\infty)}=N$ and $Z(N)=0$. Also, we have that $N / R(N)$ is isomorphic to a direct sum of supersimple Lie algebras, by the minimality of $L$. Then, by Proposition 5.6, it follows that $R(N)=Z(N)$. This yields that $N$ is semisimple and so it is simple (see [25, p. 30]). Therefore $N$ is supersimple. Then, by Lemma 5.7 it follows that $L=N \oplus C_{L}(N)$. We have that $C_{L}(N)$ is semisimple too, so it can be decomposed into a direct sum of supersimple ideals because of the minimality of $L$. We conclude that $L$ is isomorphic to a direct sum of supersimple Lie algebras, which is a contradiction. Therefore $L$ is simple, as claimed.

Next we prove that $L$ has no solvable maximal subalgebras. Let $U$ be a solvable maximal subalgebra of $L$. By Proposition 5.1(ii), we have that $U$ is the unique solvable maximal subalgebra of $L$. So, there must exist at least two nonsolvable maximal subalgebras $T$ and $T^{*}$ of $L$. Since $U \cap T$ is solvable and maximal in $T$, we see that $T / R(T)$ must be a $\mu$-algebra. Assume that $R(T)=0$. Then we see that $\operatorname{dim} T \cap U=1$. This yields $\operatorname{dim} U=2$ and hence $L \cong \operatorname{sl}(2)$ by Lemma 2.2 . But then we get $\sqrt{F} \leq F$, a contradiction. Therefore $R(T) \neq 0$. We claim that $R(T) \leq \operatorname{Fr}(L)$. Assume not. Then, there exists a maximal subalgebra $S$ of $L$ such that $R(T) \leq S$. Since $S \cap T$ is maximal in $T$, by Proposition 3.3 and Lemma 3.1 we have $T^{(\infty)} \leq S \cap T$. Thus $S \cap T$ is nonsolvable and so $S$ is nonsolvable too. This yields $R(S) \notin S \cap T$ and so $S^{(\infty)} \leq S \cap T$. But then we have $T^{(\infty)}=(S \cap T)^{(\infty)}=S^{(\infty)} \triangleleft T \vee S=L$, which is a contradiction. The claim is proved. We deduce that $R(T)+R\left(T^{*}\right)=T \cap T^{*}=\operatorname{Fr}(L)$. By Proposition 3.4(ii), $\operatorname{Fr}(L)$ is modular in $L$ and hence $\operatorname{Fr}(L)$ is self-normalizing in $L$ (see [3]). On the other hand, we have that $R(T) \cap R\left(T^{*}\right)$ is an ideal in $R(T)$ and $R\left(T^{*}\right)$. Since $\left[T^{(\infty)}, R(T)\right]=0=\left[\left(T^{*}\right)^{(\infty)}, R\left(T^{*}\right)\right]$ (Proposition 5.6), it follows that $R(T) \cap R\left(T^{*}\right) \triangleleft$ $T \vee T^{*}=L$. So, $R(T) \cap R\left(T^{*}\right)=0$. This yields $\operatorname{dim} R(T)=\operatorname{dim} R\left(T^{*}\right)=1$ and then $R(T)=Z(T)$ and $R\left(T^{*}\right)=Z\left(T^{*}\right)$. Also, we have $\operatorname{Fr}(L)=Z(T) \oplus Z\left(T^{*}\right)$. Since $\operatorname{Fr}(L)$ is 
self-normalizing and maximal in $U$ and since $U$ is supersolvable, we see that the largest ideal of $U$ contained in $\operatorname{Fr}(L)$ is just the centre of $U$. So $\operatorname{dim} Z(U)=1$. We deduce that the centre of each maximal subalgebra of $L$ has dimension one and is contained in $\operatorname{Fr}(L)$. Now we argue as in the last paragraph of the proof of Theorem 1.6 of [20]. Put $T=\operatorname{Fr}(L) \vee F x$ and $T^{*}=\operatorname{Fr}(L) \vee F y$. By Proposition 3.4, we have that $\operatorname{Fr}(L) \vee F(x+y)$ is a maximal subalgebra of $L$. So, its centre has dimension one. Pick $0 \neq z \in$ $Z(\operatorname{Fr}(L) \vee F(x+y))$. Decompose $z=u+v$ where $u \in Z(T)$ and $v \in Z\left(T^{*}\right)$. We see $u \neq 0 \neq v$. We have $0=[z, x+y]=[u, y]+[v, x]$. So, $[u, y]=[x, v] \in T \cap T^{*}=\operatorname{Fr}(L)$. This yields $[y, \operatorname{Fr}(L)]=[y, F u+F v] \leq \operatorname{Fr}(L)$ and hence $y \in \operatorname{Fr}(L)$, which is a contradiction. Consequently, $L$ has no solvable maximal subalgebras.

Now let $B$ be a solvable subalgebra of $L$ of maximal dimension. We have $\operatorname{dim} B>1$, since otherwise we would have that $L$ has no subalgebras of dimension two and hence $L$ is supersimple (see [18]), which is a contradiction. Take a maximal subalgebra $M$ of $L$ containing $B$. By the maximality of $B$, we have $R(M)<B$. We claim that $M^{(\infty)} \leq \operatorname{Fr}(L)$. Assume not. Then, there exists a maximal subalgebra $M^{*}$ of $L$ such that $M^{(\infty)} \notin M^{*}$. So $R(M) \leq M^{*}$. Since $M \cap M^{*}$ is maximal in $M$, by Lemma 5.8 we have that $M \cap M^{*}$ contains a solvable subalgebra $B^{*}$ such that $\operatorname{dim} B=\operatorname{dim} B^{*}$. This yields that $B^{*}$ is a maximal solvable subalgebra of $L$ and therefore $R\left(M^{*}\right)<B^{*} \leq M$. It follows that $R(M)+R\left(M^{*}\right) \leq M \cap M^{*}$ and so $R(M)+R\left(M^{*}\right) \leq R\left(M \cap M^{*}\right)$. Also, we have that $R(M) \cap R\left(M^{*}\right)$ is an ideal in $R(M)$ and in $R\left(M^{*}\right)$. By Proposition 5.6, $R(M) \cap R\left(M^{*}\right) \triangleleft M \vee M^{*}=L$. So, $R(M) \cap R\left(M^{*}\right)=0$. Let us now suppose that $R(M) \neq 0 \neq R\left(M^{*}\right)$. Then we see that neither $M \cap M^{*} / R(M)$ nor $M \cap M^{*} / R\left(M^{*}\right)$ is semisimple. Write $\left(M \cap M^{*} / R(M)\right)^{(\infty)}=A / R(M)$. We see $\left(M \cap M^{*}\right)^{(\infty)}=A^{(\infty)}$. By Lemma 5.8(2) we have $A \triangleleft M$. This yields $\left(M \cap M^{*}\right)^{(\infty)} \triangleleft M$. Analogously, we obtain $\left(M \cap M^{*}\right)^{(\infty)} \triangleleft M^{*}=L$. So, $\left(M \cap M^{*}\right)^{(\infty)} \triangleleft L$, which is a contradiction. Therefore either $R(M)=0$ or $R\left(M^{*}\right)=0$. Next assume that $R(M)=0=R\left(M^{*}\right)$. Then, we can decompose $M=P_{1} \oplus \cdots \oplus P_{r}$ and $M^{*}=Q_{1} \oplus \cdots \oplus Q_{s}$, where each $P_{i}$ is a supersimple ideal of $M$ and each $Q_{j}$ is a supersimple ideal of $M^{*}$. We see that $r=\operatorname{dim} B=s$, by Lemma 5.8(4). Since $M \cap M^{*}$ is maximal in $M$ and $M^{*}$, by Lemma 5.8(1) we can write

$$
M \cap M^{*}=\left(P_{1} \cap M^{*}\right) \oplus P_{2} \oplus \cdots \oplus P_{r}=\left(Q_{1} \cap M\right) \oplus Q_{2} \oplus \cdots \oplus Q_{r} .
$$

From this it follows that $r=2, P_{2}=Q_{1} \cap M$ and $P_{1} \cap M^{*}=Q_{2}$, since otherwise $M$ and $M^{*}$ would have a common nonzero ideal, which contradicts the simplicity of $L$. So $M^{*} \cap P_{1}$ is an ideal of $M^{*}$ and is maximal in $P_{1}$. Now we take a third maximal subalgebra $S$ of $L$. Since $M \cap S$ is maximal in $M$, we see that $M \cap S$ contains a solvable subalgebra of dimension two. It follows that $R(S)<M \cap S$. Let $R(S) \neq 0$. As $S \cap M$ is maximal in $M$, we have that either $P_{1} \leq S$ or $P_{2} \leq S$. If $P_{2} \leq S$, then we have $S \cap M=P_{2} \oplus\left(P_{1} \cap S\right)$ and $P_{1} \cap S=R(S)$. This yields that $R(S)$ is maximal in $P_{1}$ and hence $P_{1}$ is a $\mu$-algebra, which contradicts the fact that $Q_{2}<P_{1}$. It follows that $P_{2} \notin S$ and so $P_{1} \leq S$. Similarly, we obtain $Q_{1} \leq S$. But then we have $M=P_{1}+P_{2} \leq S$, a contradiction. Therefore $R(S)=0$. Then, $S$ (like $M$ and $M^{*}$ ) can be decomposed into a direct sum of two supersimple ideals. Assume that $Q_{1} \leq S$. Then we have $Q_{2} \leq S$ and 
so $P_{1} \notin S$. This yields that $P_{1} \cap S \triangleleft S$ and hence $P_{1} \cap S \triangleleft S \cap Q_{1}$, which contradicts the simplicity of $S \cap Q_{1}$. Therefore $Q_{1} \not S$. Similarly, we obtain $P_{1} \not S$. Hence $P_{2}+Q_{2} \leq S$. This yields $P_{1} \cap S=Q_{2}$ and hence $Q_{2} \triangleleft S \vee M^{*}=L$, which contracts the simplicity of $L$. Consequently, either $R(M) \neq 0=R\left(M^{*}\right)$ or else $R\left(M^{*}\right) \neq 0=R(M)$. Suppose $R\left(M^{*}\right) \neq 0=R(M)$. Take a third maximal subalgebra $S$ of $L$, once again. Since $R(M)=0$, we see that $M \cap S$ contains a solvable subalgebra of $L$ of maximal dimension. By the above, we have $R(S) \neq 0$. Since $R\left(M^{*}\right) \neq 0$, we find that $S \cap M^{*}$ cannot contain any solvable subalgebra of $L$ of maximal dimension. This yields that neither $R(S)$ nor $R\left(M^{*}\right)$ is contained in $S$ and therefore $S^{(\infty)}+\left(M^{*}\right)^{(\infty)} \leq S \cap M^{*}$. But then we find

$$
S^{(\infty)}=\left(S \cap M^{*}\right)^{(\infty)}=\left(M^{*}\right)^{(\infty)} \triangleleft S \vee M^{*}=L,
$$

which is a contradiction. By symmetry, the case where $R(M) \neq 0=R\left(M^{*}\right)$ cannot occur either. We conclude that $M^{(\infty)} \leq \operatorname{Fr}(L)$, as claimed.

Let $S$ be any maximal subalgebra of $L$ different from $M$. Since $M^{(\infty)} \leq S \cap M$, we have $(M \cap S)^{(\infty)}=M^{(\infty)} \triangleleft M$. This yields that $S^{(\infty)} \notin M$ and so $R(S) \leq M$. We see that $M \cap S / R(M \cap S)$ is semisimple, since otherwise by using Lemma 5.8(2) again we would obtain $(M \cap S)^{(\infty)} \triangleleft S \vee M=L$, which is a contradiction. We deduce that $R(M \cap S)=R(S)$ and $M \cap S=R(S)+M^{(\infty)}$.

Now, take a maximal subalgebra $K$ of $L$ such that $K^{(\infty)}$ is maximal. Then, we see that $K$ is the only maximal subalgebra of $L$ which contains $K^{(\infty)}$. This yields $R(K) \leq \operatorname{Fr}(L)$. By the preceding paragraph we have $R(K)+M^{(\infty)}=M \cap K=\operatorname{Fr}(L)$. Next, take a third maximal subalgebra $S$ of $L$ different from $M$ and $K$. We find $M \cap S \geq \operatorname{Fr}(L)=M \cap K$. Since $M \cap K$ is maximal in $M$, it follows $M \cap S=M \cap K$. By the preceding paragraph again, we have $R(S)=R(M \cap S)=R(M \cap K)=R(K)$. This yields $R(K) \triangleleft K \vee S=L$ and hence $R(K)=0$. Then we get $M \cap K=M^{(\infty)}$. On the other hand, by Proposition 3.4 (ii) we see that $M \cap K$ is modular in $L$. So, $M \cap K$ is selfnormalizing by [3]. This yields $M=M^{(\infty)}$. But then we have $M=\operatorname{Fr}(L)$. This contradiction completes the proof.

Now we are able to determine the structure of a lower semimodular Lie algebra in the case where $\sqrt{F} \not F$.

Theorem 5.10. Let $\operatorname{char}(F)>3$ and $\sqrt{F} \notin F$. Then, for a Lie algebra $L$ the following are equivalent:

(i) L is lower semimodular;

(ii) $R(L)$ and $L / R(L)$ are both lower semimodular,

(iii) $L / Z\left(L^{(\infty)}\right)$ is lower semimodular;

(iv) $L / Z\left(L^{(\infty)}\right) \cong U \oplus P_{1} \oplus \cdots \oplus P_{r}$, where $U$ is supersolvable, $r \geq 0$ and the $P_{i}$ are pairwise strongly non-isomorphic lower semimodular supersimple Lie algebras.

Proof. (i) $\Rightarrow$ (iv): By Theorem 5.9 we have that $L / R(L)$ is a direct sum of super- 
simple ideals. It follows that $L=R(L)+L^{(\infty)}$. By Proposition 5.6, we have $[R(L)$, $\left.L^{(\infty)}\right]=0$. This yields $R(L) \cap L^{(\infty)}=Z\left(L^{(\infty)}\right)$. By Theorem 5.9 and Lemma 5.8(5) we get that $L / Z\left(L^{(\infty)}\right)$ is as in (iv).

(iv) $\Rightarrow$ (i): Let $L$ be a counterexample of minimal dimension. Put $L / Z\left(L^{(\infty)}\right)=$ $A / Z\left(L^{(\infty)}\right) \oplus B / Z\left(L^{(\infty)}\right)$, where $A / Z\left(L^{(\infty)}\right) \cong U$ and $B / Z\left(L^{(\infty)}\right) \cong P_{1} \oplus \cdots \oplus P_{r}$. As $L$ is not lower semimodular, by Lemma 3.2 there exists a maximal subalgebra $M$ of $L$ such that $M \cap K$ is not a modular maximal subalgebra of $K$ for some maximal subalgebra $K$ of $L$ different from $M$. We see that $B=L^{(\infty)}$ and $A=R(L)$. By using Lemma 3.1 $(1,3)$ we obtain that each maximal subalgebra of $L$ contains either $A$ or $B$. If $M \geq B$, then we have $\operatorname{dim} L / M=1$ since $A$ is supersolvable. So, $\operatorname{dim} M / M \cap K=1$, which is a contradiction. Therefore $M \geq A$. Let us now suppose $K \geq A$. Then it is easy to see that $M \cap K$ is maximal in $K$. Also, we have $K=A \cap K+B, K^{(\infty)}=L^{(\infty)}=B$. This yields that the Lie algebra $K$ satisfies (iv) too. By the minimality of $L$, we have that $K$ is lower semimodular. It follows that the maximal subalgebra $M \cap K$ of $K$ is also modular in $K$, which is a contradiction. Therefore $K \geq A$. Since $L / A$ is lower semimodular (Lemma 5.8), we have that $M \cap K$ is maximal in $K$. On the other hand, we see that $K^{(\infty)} \leq L^{(\infty)}=B$ and $\left(K^{(\infty)}+A\right) / A=(K / A)^{(\infty)}$. Then, by using Lemma 5.8 we obtain $K=R(K)+K^{(\infty)}$, and $K^{(\infty)} / R(K) \cap K^{(\infty)}$ is isomorphic to a direct sum of supersimple Lie algebras which are lower semimodular and pairwise strongly nonisomorphic. Since $R\left(K^{(\infty)}\right)=R(K) \cap K^{(\infty)}$, from Proposition 5.6 it follows that $R\left(K^{(\infty)}\right)=Z\left(K^{(\infty)}\right)$. It follows that $K$ satisfies (iv) too. By the minimality of $L$, we have that $K$ is lower semimodular. This yields that $M \cap K$ is modular in $K$, which is a contradiction.

(ii) $\Rightarrow$ (iv): By Theorem 5.9 and Lemma 5.8, we have that $L / R(L)$ is isomorphic to a direct sum of pairwise strongly non-isomorphic supersimple Lie algebras. By Proposition 5.6 we have $\left[R(L), L^{(\infty)}\right]=0$. So, $R(L) \cap L^{(\infty)}=Z\left(L^{(\infty)}\right)$. Then we see that $L$ is as in (iv).

(iii) $\Rightarrow$ (ii): Put $\bar{L}=L / Z\left(L^{(\infty)}\right)$. We have $R(\bar{L})=R(L) / Z\left(L^{(\infty)}\right)$. So, $\bar{L} / R(\bar{L}) \cong L / R(L)$. Since $\bar{L}$ is lower semimodular, so are $\bar{L} / R(\bar{L})$ and $R(\bar{L})$. Thus $L / R(L)$ is lower semimodular and $R(L) / Z\left(L^{(\infty)}\right)$ is supersolvable. Since $Z\left(L^{(\infty)}\right) \leq \phi(L)$ (Lemma 3.1(1)), by [5] it follows that $R(L)$ is supersolvable. So $R(L)$ is lower semimodular too. Clearly, (i) implies (ii) and (iii). The proof is complete.

We recall that if the quotient of a Lie algebra $G$ by its centre is three-dimensional simple, then $G=Z(G) \oplus G^{\prime}$ (see [20]). We wonder if the same holds when $G / Z(G)$ is supersimple. If it does, then we would obtain that $Z\left(L^{(\infty)}\right)=0$ whenever $L$ is lower semimodular and $\sqrt{F} \notin F$ arguing as in the proof of the following.

Corollary 5.11. Let $F$ be perfect with $\operatorname{char}(F)>3$ and $\sqrt{F} \notin F$. Then, a Lie algebra $L$ is lower semimodular if and only if $L=U \oplus P_{1} \oplus \cdots \oplus P_{r}$ where $U$ is a supersolvable ideal of $L, r \geq 0$ and the $P_{i}$ are mutually non-isomorphic three-dimensional non-split simple ideals of $L$.

Proof. By our assumption on the ground field, we have that the three-dimensional 
non-split simple algebras are the only Lie algebras which are supersimple (see [19] and [10]). Assume that $L$ is nonsolvable and lower semimodular. Then, by Theorem 5.10 we can decompose $L^{(\infty)} / Z\left(L^{(\infty)}\right)=P_{1} / Z\left(L^{(\infty)}\right) \oplus \cdots \oplus P_{r} / Z\left(L^{(\infty)}\right)$, where each $P_{i} / Z\left(L^{(\infty)}\right)$ is three-dimensional non-split simple. We see that $Z\left(L^{(\infty)}\right)=Z\left(P_{i}\right)$, for every $1 \leq i \leq r$. Then we have $P_{i}=Z\left(L^{(\infty)}\right) \oplus P_{i}^{\prime}$ (see [20]). This yields

$$
L^{(\infty)}=P_{i}^{\prime} \oplus \cdots \oplus P_{r}^{\prime} \oplus Z\left(L^{(\infty)}\right) \text {. }
$$

As $\left(L^{(\infty)}\right)^{(\infty)}=L^{(\infty)}$, it follows that $Z\left(L^{(\infty)}\right)=0$. Now, the result follows from Theorem 5.10.

Corollary 5.12. Let $F$ be finite with char $(F)>3$. Then, the supersolvable algebras are the only Lie algebras which are lower semimodular.

Proof. It is well-known that there are no three-dimensional non-split simple Lie algebras over $F$ (see [8]). So, the result follows from Corollary 5.11.

Acknowledgment. The author is grateful to A. G. Gein for useful discussions.

\section{REFERENCES}

1. R. K. Amayo, Quasi-ideals of Lie algebras I, Proc. London Math. Soc. 33 (1976), 28-36.

2. R. K. Amayo, Quasi-ideals of Lie algebras II, Proc. London Math. Soc. 33 (1976), 37-64.

3. R. K. Amayo and J. Schwarz, Modularity in Lie algebras, Hiroshima Math. J. 10 (1980), 311-322.

4. D. Barnes, On Cartan subalgebras of Lie algebras, Math. Z. 101 (1967), 350-355.

5. D. Barnes and M. L. Newell, Some theorems on saturated homomorphs of soluble Lie algebras, Math. Z. 115 (1970), 179-187.

6. G. Benkart, I. M. IsAaCs and J. M. Osborn, Lie algebras with self-centralizing ad-nilpotent elements, J, Algebra 57 (1979), 279-309.

7. G. Benkart and J. M. Osborn, Rank One Lie algebras, Ann. of Math. (2) 119 (1984), 437-463.

8. R. Farnsteiner, Ad-semisimple Lie algebras, J, Algebra 83 (1983), 510-519.

9. A. G. Gein, Semimodular Lie algebras, Sibirsk. Mat. Zh. 17 (1976), 243-248; (translated in Siberian Math. J. 17 (1976), 189-193).

10. A. G. GeIN, Modular rule and relative complements in the lattice of a Lie algebra, Izv. Vyssh. Uchebn Zaved. Mat. 383 (1987), 18-25; (translated in Soviet Math. 331 (1987), 22-32).

11. A. G. Gein, On modular subalgebras of Lie algebras, Ural Gos. Univ. Mat. Zap. 14 (1987), 27-33.

12. N. JACOBSON, Lie algebras (New York: Wiley, Interscience 1962).

13. H. StRAde and R. FARnsteIner, Modular Lie algebras and their Representations. (Textbooks and Monographs, vol. 116, New York: Dekker 1988). 
14. D. A. Towers, A Frattini theory for algebras, Proc. London Math. Soc. 27 (1973), $440-462$.

15. D. A. TOWERs, Lie algebras all of whose maximal subalgebras have codimension one., Proc. Edinburgh Math. Soc. 24 (1981), 217-219.

16. D. A. Towers, On ideally finite Lie algebras which are lower semimodular, Proc. Edinburgh Math. Soc. (2) 28 (1985), 9-11.

17. V. R. Varea, Lie algebras whose maximal subalgebras are modular, Proc. Roy. Soc. Edinburgh Sect. A 94 (1983), 9-13.

18. V. R. VAREA, Lie algebras none of whose Engel subalgebras are in intermediate position, Comm. in Algebra 15(12) (1987), 2529-2543.

19. V. R. VAREA, The subalgebra lattice of a supersolvable Lie algebra, in Lie algebras (Madison 1987, Springer Lectures Notes in Math. 1373, 1989), 81-92.

20. V. R. VAREA, On modular subalgebras in Lie algebras of prime characteristic, Contemp. Math. 110 (1990), 289-307.

21. V. R. VAREA, Modular subalgebras, quasi-ideals and inner ideals in Lie algebras of prime characteristic, Comm. in Algebra 21(11) (1993), 4195-4218.

22. V. R. VAREA, Lie algebras whose proper subalgebras are either semisimple, abelian or almost-abelian, Hiroshima Math. J. 24 (1994), 221-241.

23. V. R. VAREA, Lie algebras all of whose proper subalgebras are solvable, Comm. Algebra 23(9) (1995), 3245-3267.

24. V. R. VArea, Supersimple and upper semimodular Lie algebras, Comm. Algebra 23(6) (1995), 2323-2330.

25. D. Winter, Abstract Lie Algebras (MIT Press, Cambridge, Mass., 1972).

26. D. J. WinTER, Cartan Decompositions and Engel subalgebra Triangulability, J. Algebra 62 (1990), 400-417.

Department of Mathematics

UNIVERSITY OF ZARAGOZA

50009 ZARAGOZA

SPAIN

E-mail address: varea@posta.unizar.es 